\title{
Sistemas de usuario en la gestión online de contenidos biomédicos
}

\author{
Por Toni González-Pacanowski y Pablo Medina-Aguerrebere
}

\begin{abstract}
Resumen: Las demandas informativas de los ciudadanos sobre la salud han llevado a varias organizaciones a elaborar sistemas personalizados de autogestión de la información médica que facilitan la administración de esta información a ciudadanos, profesionales de la salud, instituciones públicas, empresas y grupos de pacientes. Gracias a las nuevas tecnologías, el paciente asume un rol activo que se plasma en la consulta en internet de distintos aspectos relacionados con la salud, lo que hace surgir un debate sobre las acreditaciones de los sitios web que difunden información sanitaria. En este marco, Google, con las aplicaciones Google Health y Flu Trends; y Microsoft, con la utilidad Microsoft HealthVault, se convierten en los máximos exponentes de los gestores de información médica.
\end{abstract}

Palabras clave: Autogestión, Información, Salud, Tecnología, Internet.

\section{Title: User systems in online management of bi- omedical content}

Abstract: Public demand for information about health matters has led several organizations to develop custom systems for self-management of medical information. These systems facilitate the management of this information by the public, health care professionals, public institutions, companies and groups of patients. Thanks to new technologies, patients are taking an active role in searching the internet with queries about health-related issues, which gives rise to a debate about the accreditations of web pages that disseminate health information. In this context, Google, with applications such as Google Health, Flu Trends and Android, and Microsoft, with its HealthVault utility, have become the maximum exponents of medical information management software.

Keywords: Self-management, Information, Health, Technology, Internet.

González-Pakanowski, Toni; Medina-Aguerrebere, Pablo. "Sistemas de usuario en la gestión online de contenidos biomédicos". El profesional de la información, 2010, mayo-junio, v. 19, n. 3, pp. 260-268.

DOI: 10.3145/epi.2010.may.06

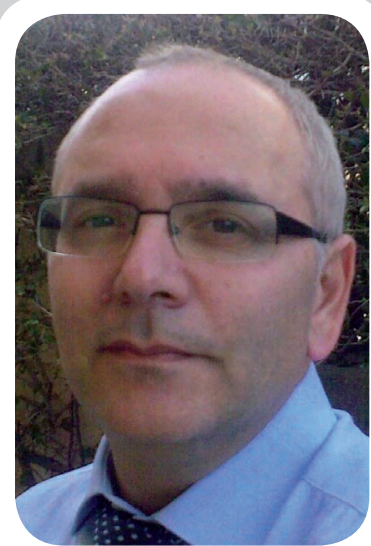

Toni González-Pacanowski es doctor en comunicación por la Univ. Pompeu Fabra (UPF) y profesor de Tecnología de la Información y Producción Periodística de la Univ. CEU Abat Oliba de Barcelona. Experto en comunicación interactiva y consultor de nuevas tecnologías en el IESE Business School de Barcelona. Colabora con DigiDoc de la UPF y con el Observ. Comunicación y Salud de la Univ. Autònoma de Barcelona. Es co-autor del libro "Web semántica y sistemas de información documental" (ed. Trea) con un capítulo dedicado a salud y medicina.

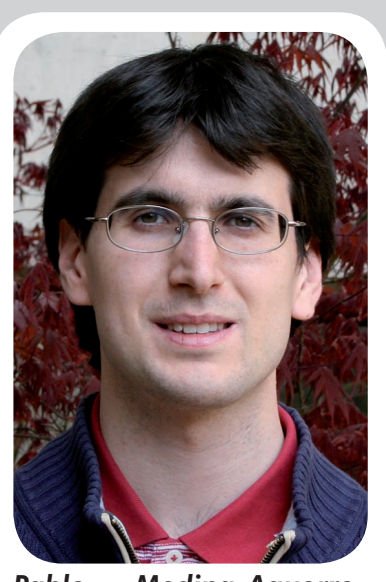

Pablo Medina-Aguerrebere es licenciado en publicidad y relaciones públicas por la Universidad de Navarra, diploma en Dirección de Cuentas Publicitarias por la Escuela Superior de Publicidad de París, y diploma de Estudios Avanzados por la Universidad de Navarra. Ha trabajado cuatro años en el sector de la comunicación de salud y actualmente prepara un doctorado sobre este tema en la citada Universidad de Navarra, donde además ejerce labores docentes en el campo de la comunicación institucional y del marketing

\section{Introducción}

El aumento de las necesidades sanitarias conlleva un incremento de la demanda de información hipersegmentada y centrada en consultas cada vez más específicas.

Factores como las nuevas posibilidades de la genómica y la tecnología sanitaria de última generación, la aparición de tratamientos cada vez con mejor costeefectividad y la confirmación de que la población mundial es susceptible de padecer una pandemia suponen

Artículo recibido el 20-1 1-09

Aceptación definitiva: 08-02-10

un aumento progresivo del uso de internet como fuente informativa y canal de comunicación.

El fenómeno de la sociedad sobre-informada con altas dosis de infoxicación es un hecho en el caso de la salud y de la medicina. Internet convierte en iguales a expertos y a otros que no lo son, y aloja a «especialistas» que supuestamente están en condiciones profesionales y legales de atender a pacientes y usuarios de la sanidad. Bajo un sello de calidad privado exhibido en un banner, como el HonCode que concede la Health on 
the Net Foundation de Suiza ${ }^{1}$, puede existir cierto compromiso ético, pero esto no evita el fraude o la mala praxis, ni siquiera acredita para el ejercicio de la medicina ni garantiza los derechos de los pacientes. Es más, este tipo de sellos de acreditación, supuestamente independientes y profesionales, pueden desnaturalizarse y convertirse más en un elemento de imagen de marca y credibilidad comercial que en una garantía. La reputación y el prestigio de la atención médica siguen siendo el sello distintivo en internet y en cualquier medio.

En este entorno de riesgo informativo y documental, la llegada de los sistemas de autogestión de la información médica personalizada y monitorizada se produce en el entramado de una sociedad que recurre a su propia fuerza cognitiva y experimental para compartirla en red. Al mismo tiempo, la sociedad busca y selecciona fuentes acreditadas y con reputación suficiente para formarse una opinión sobre su estado o sobre su problema de salud específico. Unido a este proceso, la percepción del propio estado de salud conduce a confeccionar entornos de comunicación interactivos colaborativos por tratarse de necesidades esenciales en la vida del individuo. Incide en todo ello el perfil del ciudadano que pertenece a colectivos desfavorecidos, con dificultades para entrar en los servicios sanitarios y que obtiene en internet un medio para compensar, al menos parcialmente, el déficit de atención médica presencial y convencional.

\section{"Las redes de información médica pueden ser profesionales, institucionales, comerciales o sociales"}

\section{Modelos de red informativa}

Podríamos hablar de cuatro grandes redes de información médica según su tipo de audiencia, velocidad de implantación y naturaleza de los contenidos. Éstas serían la profesional, institucional, comercial y social.

\section{Profesional}

Tiene sus canales constituidos y está en proceso de perfeccionamiento. Está vinculada a instituciones sanitarias, editoriales de prestigio, bases de datos y empresas interesadas en el segmento de la audiencia científica y profesional. Algunos exponentes son Medline en el terreno público y la editorial Elsevier. Si bien su potencial documental es enorme, por el momento no se encuentran directamente relacionadas con sistemas de autogestión de la información médica personalizada.

http://www.ncbi.nlm.nih.gov/pubmed/

http://www.elsevier.com/wps/find/homepage.cws_home
Éstas y otras fuentes que utilizan los profesionales están disponibles para el público y por tanto, constituyen un motor de activación de la propia red social que la utiliza para confirmar e intercambiar información. Según Brownstein, Freifeld y Madoff (2009), algunas de estas fuentes de libre consulta y que los portales de autogestión de la información médica pueden llegar a utilizar son las siguientes:

- Promed-mail

http://www.promedmail.org

- Global public health intelligence network (Gphin) http://www.phac-aspc.gc.ca/media/nr-rp/2004/2004_ gphin-rmispbk-eng.php

- Healthmap

http://www.healthmap.org

- Medisys

http://medusa.jrc.it

- Epispider

http://www.epispider.org

- Biocaster

http://biocaster.nii.ac.jp

- Wildlife disease information node http://wildlifedisease.nbii.gov/

- H5N1 Google Earth mashup http://www.nature.com/avianflu/google-earth

- Avian influenza daily digest and blog http://influenzadigest.net/news/

- Google Flu Trends

http://www.google.org/flutrends

- Google insights for search http://www.google.com/insights/search

- Distribute

http://www.syndromic.org/projects/distribute.htm

- Geosentinel

http://www.istm.org/geosentinel/main.html

- Emerging infections network http://ein.idsociety.org

- Aarhus

http://biodefense.georgetown.edu

\section{Institucional}

Estructurada directamente desde los entes gubernamentales, está dirigida al público, pero con una clara intención de control y supervisión sobre la información personalizada. Su evolución se encuentra condicionada por la exigencia de cumplir con fines socio-sanitarios y de alcanzar fronteras donde es preciso atender requerimientos sociales y profesionales sanitarios. 
En el entorno español, encontramos algunos ejemplos como el Servicio Gallego de Salud (Sergas), en fase piloto el Servicio Catalán de Salud (SCS) y experimentalmente en varios países europeos. En el caso del Servicio Gallego de Salud, el navegante accede a una oficina virtual para tramitar gestiones mediante certificado digital. La interactividad consiste en que los usuarios pueden enviar sugerencias y reclamaciones. Igualmente, dispone de un servicio de canales RSS temáticos, sistema de actualización de noticias, avisos, alertas farmacéuticas y publicaciones de este organismo público.

La llamada e-salud de las administraciones sanitarias se extiende en función de su propia infraestructura con una oferta de contenidos y servicios, pero limitada para el ciudadano en aspectos colaborativos sociales. Se trata de sistemas asistenciales que utilizan las nuevas tecnologías y las ponen al servicio del ciudadano y de los profesionales, aportando soluciones tanto estáticas como para dispositivos móviles.

\section{Comercial}

Tiene sus máximos exponentes en Google y $\mathrm{Mi}$ crosoft, cuyos sistemas guardan similitudes. Su avance está limitado por el marco normativo sanitario de cada país, lo que conlleva que sólo las personas autorizadas pueden beneficiarse. Si bien pueden cumplir una función asistencial, no son empleadas como tales en el ámbito institucional. El hecho de que se considere aquí a Google Health como una red comercial a pesar de ser de libre acceso, es porque su viabilidad depende de colaboradores empresariales para su supervivencia. El debate en torno a si el ciudadano puede manipular su propia información clínica o recopilar determinados resultados que su médico de cabecera prohíba está presente en estas aplicaciones, del mismo modo que la información sobre las garantías de la confidencialidad y la conformidad expresa del usuario (Tang; Lee, 2009).

http://www.google.com/health

http://www.healthvault.com/

\section{"El ciudadano cada vez consulta más información de salud en internet"}

\section{Social}

La conforman los grupos de autoayuda de pacientes, así como las iniciativas individuales, foros, blogs y aplicaciones donde no hay un control institucional o profesional directo. Su rasgo distintivo es que es colaborativa, vinculada estrictamente a la sociedad civil.
Así, destacan portales como New Hampsire citizens for health freedom que cuestionan la medicina convencional, y otros como los foros de pacientes, los grupos de autoayuda e incluso los grupos de experiencias personales de gran valor moral. Una nota diferencial es que estos colectivos han trasladado su estrategia de acción social a la Red, constituyendo dispositivos de reivindicación política, campus online y sistemas de documentación compartidos, entre otros servicios.

http://www.nhhealthfreedom.org/choice.htm http://www.cuentanostuplancontralaleucemia.blogs pot.com/

http://www.webpacientes.org/fep/

En el punto de intersección entre estos modelos básicos está, por ejemplo, el caso de Patientslikeme, donde la colaboración es el eje del contenido y donde encontramos como patrocinadores tanto entidades altruistas, como laboratorios farmacéuticos o servicios de centros hospitalarios que proporcionan al paciente información de máxima calidad y le ofrecen la oportunidad de entrar en contacto puntual con su cuadro médico. En esta clasificación influye la denominada brecha digital, con notable influencia del factor edad. En este sentido, el nivel de envejecimiento de la población europea incide en el distanciamiento y en la reticencia al uso de la e-salud. Aunque hablemos de una renovación generacional y de una progresiva alfabetización digital, la incapacidad de adquirir tecnologías de última generación o de renovarlas, el factor geográfico o la misma implantación de la banda ancha son constantes que sin duda influyen en el nivel de disposición y de uso de la información médica en internet.

http://www.patientslikeme.com/

\section{Progresión creciente del uso}

La estadística demuestra que las consultas en internet sobre salud y medicina se incrementan progresivamente en las sociedades avanzadas, a la vez que se dispara el uso de las redes sociales relacionadas con el mundo sanitario. La progresión se mantendrá durante los próximos años con el techo puesto en el momento en que la mayor parte de la población pueda conectarse a internet.

Las fuentes que se presentan a continuación miden el comportamiento y alcanzan a determinar los intereses de la audiencia, categorizan la importancia del consejo médico y familiar frente al hecho único de la consulta del material y dan a entender cuáles son sus niveles de networking en la relación con los fondos sanitarios.

En España encontramos algunos datos procedentes del Instituto Nacional de Estadística (INE) y de entidades privadas. El INE (2009), en su última Encuesta sobre equipamiento y uso de tecnologías de la infor- 
mación y comunicación en los hogares realizada entre 2007 y 2008, refleja un aumento de la búsqueda de información sobre temas de salud. Los usuarios controlados pasan de un 41,4 a un 43,7\%. A día de hoy más de 8,5 millones de personas buscan información sobre aspectos de la salud.

El Departamento de Estudios Sociales y Opinión Pública de la Fundación BBVA (2008), en su II Estudio sobre internet en España, también muestra un aumento de más del $11 \%$ con respecto a la encuesta del año 2005: cerca de un $35 \%$ de la población ha utilizado la Red para conseguir información sanitaria. Se señala que al menos un $14 \%$ de los encuestados no se plantea siquiera si la información a la que accede en internet es fiable, aunque una parte significativa espera encontrar información fiable en asuntos de salud (puntuación de 7,2 sobre una escala de 10$)$.

En Estados Unidos, y si se toma como referencia el análisis de Pew \& American Life Project y California HealthCare Foundation (2009), destaca que el $61 \%$ de los adultos ha hecho búsquedas sobre información de salud. De este colectivo, el 59\% ha realizado una de las siguientes actividades:

- Lectura de comentarios en servicios de noticias en línea, sitios web o blogs

- Consulta de rankings de médicos o de otros profesionales sanitarios.

- Consulta de rankings de hospitales y otras instalaciones sanitarias.

- Consentimiento para recibir novedades sobre aspectos de salud.

- Escucha de podcasts sobre salud o medicina.

Es curioso que el $20 \%$ de las personas que utilizan internet, una vez implicados en la búsqueda, hayan contribuido activamente con comentarios y opiniones, bien en blogs, en foros o en otros formatos similares.

El informe es un buen botón de muestra para corroborar que la transformación del perfil del paciente pasivo en paciente proactivo es un hecho y está convirtiendo a este colectivo en un complemento de las fuentes tradicionales de información. Son generadores de experiencias que hasta ahora estaban fuera de las fórmulas habituales para documentar la casuística clínica, pero que ayudan de forma eficaz en la configuración de materiales de gran utilidad. Además, se constata que este contenido social que crece entre el uso de blogs, podcasts y otros medios en línea tendrá que ser valorado y gestionado por gestores de comunidades.

La conversión de estas aplicaciones se podría concretar en si realmente se percibe un beneficio para la salud. El $60 \%$ de los e-pacientes consideró que la infor- mación fue práctica. Esto representa un aumento significativo respecto al informe de 2006 del Pew \& American Life Project donde el porcentaje de e-pacientes que consideraba como práctica la información era del $31 \%$. Si se observa el nivel de riesgo como un indicador, se obtiene que el $3 \%$ se consideró perjudicado por información de salud o por consejos médicos en la Red, un nivel de incidencias que no ha variado desde el 2006. Otra conclusión de la última oleada del Pew \& American Life Project indica que internet está en tercer lugar como fuente de información médica (empatado con la consulta de libros), por detrás de las consultas a profesionales de la salud y las conversaciones con amigos o familiares. El tipo de consultas que han ganado cuota en las búsquedas de información son las siguientes:

- Enfermedad o complicación médica.

- Tratamiento o procedimiento médico.

- Cómo estar en forma.

- Prescripción o medicamentos de venta libre.

- Tratamientos alternativos o medicamentos.

- Depresión, ansiedad, estrés o problemas de salud mental.

- Tratamientos experimentales o medicamentos.

- Métodos para bajar de peso o controlar el mismo.

- Consejos sobre cómo gozar de buena salud en un viaje al extranjero.

Según Moyá-Garrido (2009), el uso de la e-health es frecuente para aprender hábitos saludables y buscar información tras el diagnóstico, siendo los campos de la salud de más interés las dietas y el control de peso, las alergias, la obesidad y el cáncer. Lógicamente, la medicación, en lo que se refiere a los efectos secundarios y a la seguridad, también forma parte de los asuntos estrella consultados. Entre sus conclusiones, destaca que, entre la población mundial, internet es la primera fuente de información sobre salud, por delante de la consulta a profesionales sanitarios y de la consulta de libros.

En este escenario de máximo riesgo para los usuarios de internet, han surgido opciones de autogestión de la propia información que facilitan la selección de contenidos de calidad, así como colectivos estructurados que capacitan el intercambio de datos y opiniones sobre patologías. Son fenómenos que no responden a la estrategia institucional y que, muy posiblemente, sean precisamente la alternativa a las deficiencias de la sanidad pública en sus materiales online. Hoy, los servicios de autogestión de información y de servicios sanitarios digitales son pasivos y se limitan, en la gran mayoría de casos, a la solicitud de cita previa, aunque bien es cierto 


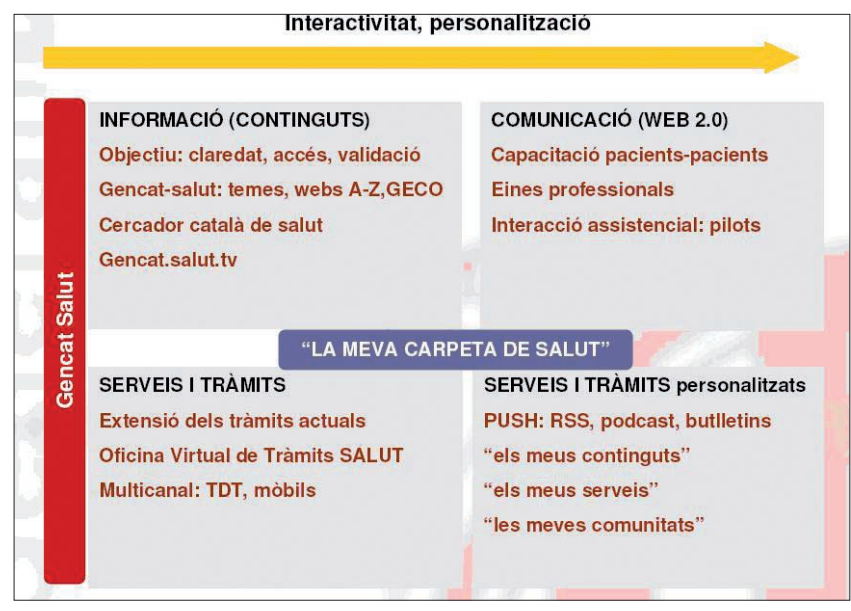

Figura 1. La receta electrónica en Cataluña

que hay previstas intranets que permitirían incluso la consulta del historial clínico por parte del paciente y la adquisición y dispensación de recetas electrónicas.

Según Lupiáñez-Villanueva (2009, p. 321), “internet se constituye como espacio de difusión de información, mientras que como espacio de interacción, con la excepción del correo electrónico, aún está infrautilizado". En el caso de Cataluña, se considera la puesta en marcha de la integración de un canal de salud para los ciudadanos y de estaciones de trabajo para los clínicos. El esquema que aquí se expone (figura 1) muestra cómo se intentan relacionar servicios y trámites con información validada consultable por el ciudadano (Guanyabens, 2009).

Evidentemente, y vistos los resultados de la llamada tarjeta sanitaria, este método puede acabar con las limitaciones de comprobación y de atención ciudadana que, en muchos casos, es perjudicada por los excesos de burocracia. Para los sistemas de salud supone avances, optimización de procesos en la comunicación entre profesionales y planificación del gasto farmacéutico y sanitario en general.

El proyecto que se centra en la historia clínica compartida ha seguido dos líneas de trabajo coordinadas en el denominado SNS proyecto piloto de interoperabilidad: historia clínica electrónica española y el Proyecto SOS (Smart Open Services) de la Unión Europea. Un caso muy diferente es el de las redes de la telemedicina avanzadas, enfocadas al uso interhospitalario.

Sin embargo, la escasa implantación de la tecnología digital en muchos lugares y la todavía limitada y encarecida banda ancha de internet son obstáculos inevitables, sin contar el escaso conocimiento que sobre el tema tienen los sanitarios y las mismas personas que utilizan estos programas. Además, esta implantación, que entraña la necesidad de aunar procedimientos tecnológicos, arquitecturas de contenido y documentación, es toda una incógnita, con un presupuesto astronómico y con el riesgo de la manipulación de información confidencial de los pacientes. La frontera de este propósito ambicioso que cambiaría la vida digital de los pacientes es el año 2012 en el caso de las administraciones sanitarias españolas.

\section{"Microsoft tiene dos opciones de uso de la autogestión online de la salud: Microsoft Health Vault y el portal MSN"}

\section{Escenario en evolución}

Google Health se ha convertido precisamente en un caso que rompe la visión estática de la gestión pública de la medicina en internet. El modelo estratégico viene a establecer una alianza con diversos proveedores de contenido, entre ellos la Cleveland Clinic de Ohio (EUA), pero dando siempre al usuario el control sobre la consulta y la selección de sus fuentes. Las intenciones de Google con la medicina son claras: suministrar aparatos de vigilancia y de autocontrol de la enfermedad con el apoyo y ayuda de centros de referencia y de bases de datos prestigiosas. En la arquitectura de este portal sólo faltan funciones más de tipo blog y aplicaciones que automaticen cálculos clínicos así como programas informáticos que generen de modo automático informes para el personal médico.

\subsection{Flu Trends, la gran innovación}

Google ha encontrado en la medicina un filón interesante. De hecho ha lanzado en su portal todo un símbolo de lo que puede representar la colaboración en línea de millones de personas en el seguimiento de una enfermedad: el ejemplo más claro es su Flu Trends (figura 2) que hizo posible visualizar la evolución de la gripe A en todo el mundo.

\section{http://www.google.org/flutrends/intl/en_gb/}

Esta iniciativa se examina en la revista Nature, que concluye la correlación entre la incidencia de la enfermedad y la frecuencia de consulta mediante el buscador Google, lo que sería útil para pensar en estimaciones de la evolución posible de la enfermedad y de las tendencias de la incidencia (Ginsberg; Mohebbi; Patel; Brammer; Smolinski; Brilliant, 2009). Otros autores han confirmado la necesidad de utilizar Google Trends, que trabaja igualmente con el sistema estadístico basado en búsquedas de Google para la prevención y recogida de información epidemiológica precisamente por su capacidad predictiva (Carneiro; Mylonakis, 2009). El aparato estadístico se basa en el volumen de búsquedas relacionadas con $f l u$ (gripe) y con palabras 


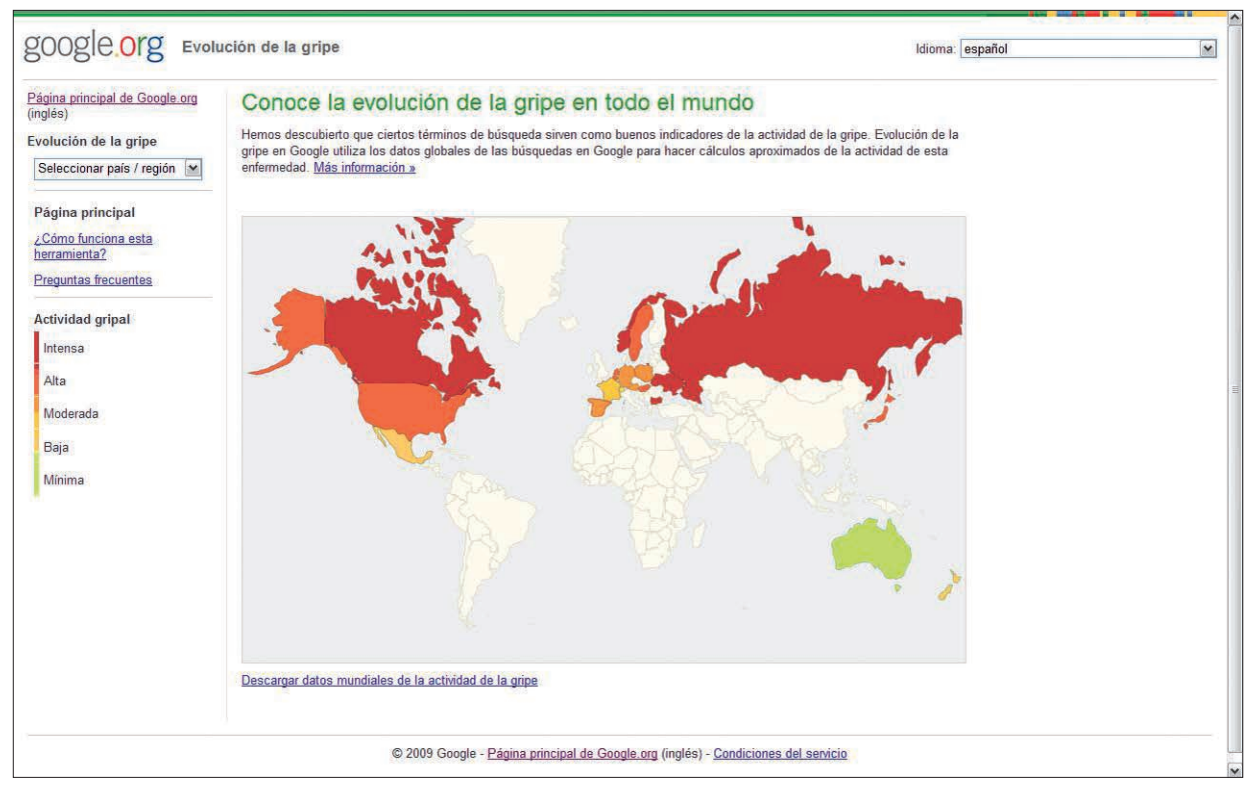

Figura 2. Seguimiento del progreso de una enfermedad en el mundo

de las mismas IPs, las tasas de rebote (visitas rápidas de sólo una página) y el tráfico generado por dispositivos móviles cuyo perfil de usuario no es estático y, por tanto, no se puede atribuir fácilmente a un país o región. Google, por el momento, está interesada en investigar si el procedimiento utilizado para detectar la actividad de la gripe puede funcionar con otras enfermedades.

Estamos, por tanto, ante una auténtica revolución de las fuentes informativas que puede superar incluso a las sanitarias oficiales. En el

clave relacionadas y, en algunos países, se basa en los datos oficiales de la vigilancia epidemiológica. Genera información de cada país de forma instantánea y en formato multilingüe y filtrado permitiendo conocer en tiempo real los resultados, sin necesidad de mediar ninguna confirmación o acreditación oficial médica.

Según informa la misma empresa en la Red, se trata de estimaciones sobre la acción de la gripe, tomando como referencia consultas globales en el buscador. Éstas se someten a un proceso de verificación comparando con datos históricos oficiales que, a su vez, se han recogido de países con vigilancia. Para los países que no proporcionan esta información, se opta por catalogarlos como "experimental". Si bien se trata de una fase piloto y las estimaciones calculadas según búsquedas comparadas con la información epidemiológica clínica oficial todavía presentan diferencias, es posible que se vayan corrigiendo los desfases.

\section{"El futuro de la autogestión de contenidos biomédicos pasa por las tecnologías móviles"}

Una de las consecuencias que entrañan estas iniciativas es la diferenciación entre el tráfico de consulta natural y el artificial, según la intención del usuario. Es decir, qué volumen de búsquedas es realmente por interés o necesidad del afectado o enfermo potencial y qué volumen corresponde con el de simples navegantes cuya intención no es estrictamente sanitaria. Igualmente, debería constar el volumen de consultas repetidas caso de la gripe, con la aplicación de Google en España, según estimaciones de analistas de la compañía líder de distribución de dominios Arsys, se contabilizaron más de 200.000 búsquedas del término gripe $A$ / H1N1 en los primeros cuatro días desde que se detectó la enfermedad. Las personas que acceden a los blogs generaron más de 1.600 comentarios. A escala mundial y en el contexto micro-blogging, cada segundo se registraba un comentario en Twitter.

http://www.arsys.info/dominios/health-un-dominiopara-garantizar-la-calidad-del-contenido-sanitarioen-la-red/

Es por tanto la misma población la que se beneficia mediante el uso de la tecnología. Sus reacciones son, lógicamente, el mayor uso y búsqueda de información específica y la demanda de servicios sanitarios. Sin embargo, el servicio que proporciona Google para gestionar la propia información médica, desde el historial clínico hasta todo tipo de opciones para compartir en línea la identidad sanitaria (figura 3), parece haber encontrado los límites en los marcos legales de numerosos países donde no es posible utilizar esta información como válida y estandarizada para los servicios sanitarios. De hecho, el programa todavía no está traducido y previsiblemente se expanda especialmente entre ciudadanos de Estados Unidos y Canadá, cuya legislación es más flexible.

\subsection{Competir con Microsoft y Yahoo}

El sistema de Google parece estar correctamente orientado, con fuentes de máxima calidad como la revista Drugs y la Cleveland Clinic. Pero Microsoft ha reaccionado con una iniciativa muy potente para el cliente basada en una alianza con la Mayo Clinic de Rochester (EUA). Microsoft HealthVault (figura 4) aporta una usa- 
bilidad más razonada, menos open source y más intuitiva, pero conlleva registros previos y discrimina todavía diversos países por motivos legales. De la misma forma que Google Health, ayuda a almacenar la información médica individual, a recibir orientación y recomendaciones basadas en la experiencia clínica de la Mayo Clinic, y amplía las capacidades interactivas de los tradicionales registros personales de salud, utilizando la información personalizada para generar recomendaciones.

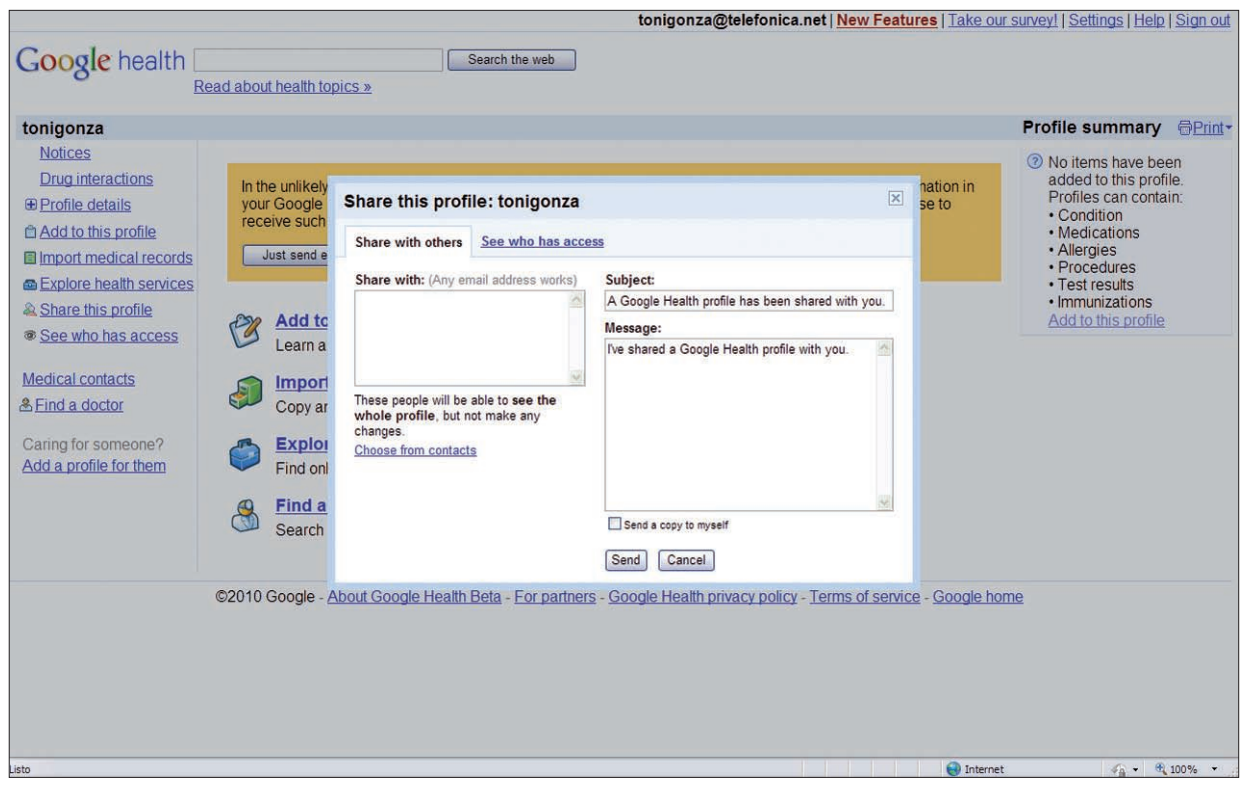

Figura 3. Aplicación de Google para administrar información médica
La limitación de Micro-

soft en el área de la salud interactiva es su escasa capacidad para poner a disposición de la gran audiencia tecnologías de libre acceso en tiempo real, como sucede en el caso del medidor de búsquedas sobre gripe. Se podría entender que esta realidad responde a su estrategia de dar pasos sólo bajo la seguridad de la captación de datos personales, la consecución de nuevas audiencias y el establecimiento de alianzas con fuentes interesadas en mejorar su reputación online mediante Microsoft. Considerado desde el punto de vista comercial, Microsoft es uno de los principales proveedores del sector sanitario en sistemas de administración clínica y, obviamente, su intención no es regalar a millones de clientes la clave de su negocio. En cualquier caso es loable su apuesta por el cuidado de la población en momentos de pandemia y su interés en que los usuarios registrados puedan disfrutar de estos servicios en sus dispositivos.

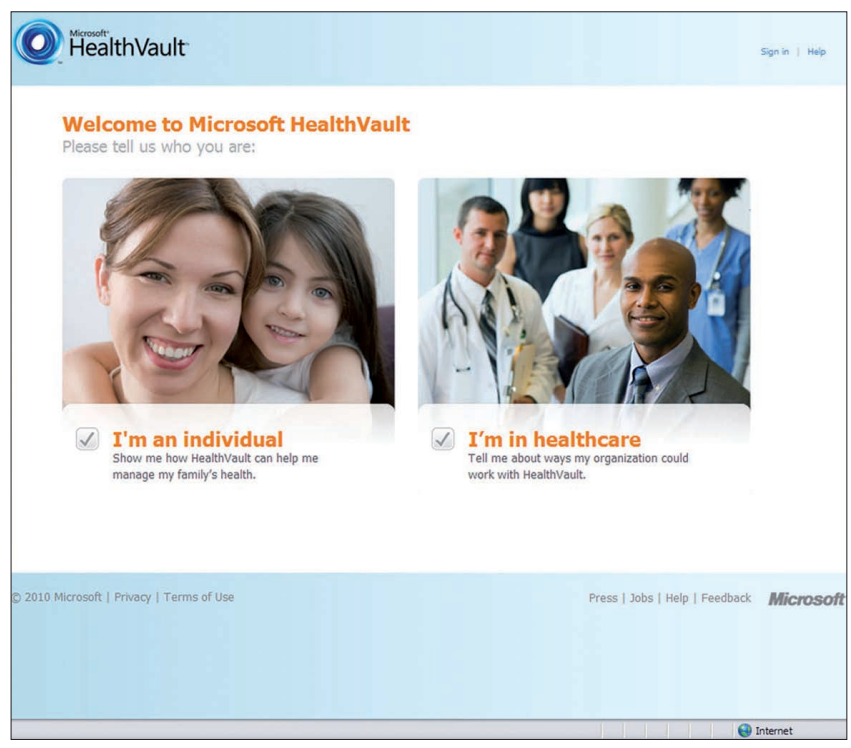

Figura 4. Página principal de Microsoft HealthVault
A diferencia de Google, Microsoft mantiene abiertas dos vías de uso de la autogestión digital de la salud, bien desde Microsoft HealthVault, con opción de utilidades para el profesional; o mediante el conocido portal de $M S N$ (figura 5), más amigable, comercial y dirigido al bienestar y a la calidad de vida.

\section{http://www.healthvault.com/ \\ http://health.msn.com/my-health-info-center/}

Yahoo, cuya estrategia se orienta a perfeccionar la potencia de sus aplicaciones y buscadores, todavía no se ha estrenado en esta área y apuesta por el conocimiento colaborativo en línea mediante sus foros basados en el sistema de pregunta-respuesta (figura 6). Es evidente que se trata de una alternativa ágil, directa y sin interferencias de posibles proveedores interesados, pero muy arriesgada, dado que la información no está sometida a ninguna revisión ni se dispone de la posibilidad de retirarla por suponer un riesgo para la salud.

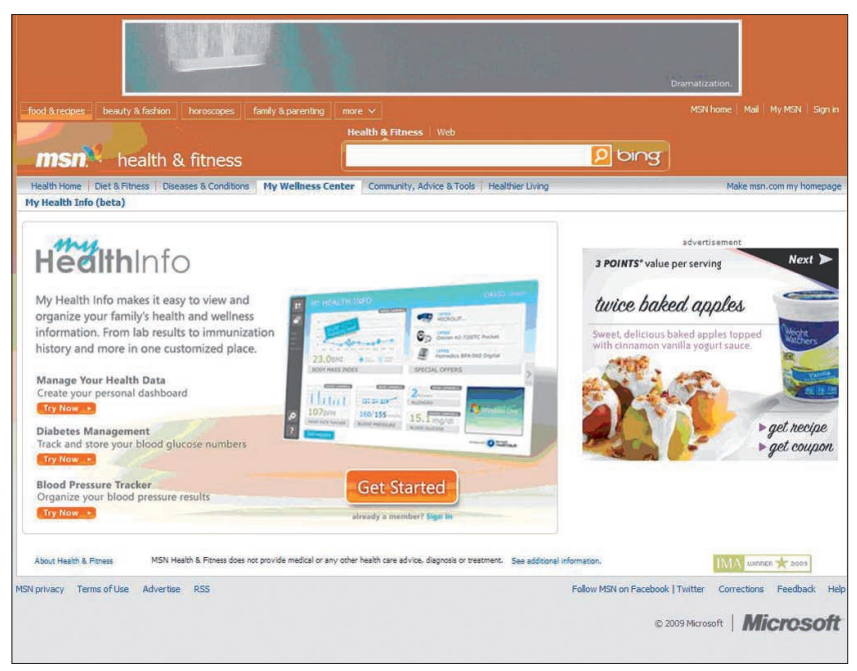

Figura 5. Página de inicio de MyHealth info (MSN) 


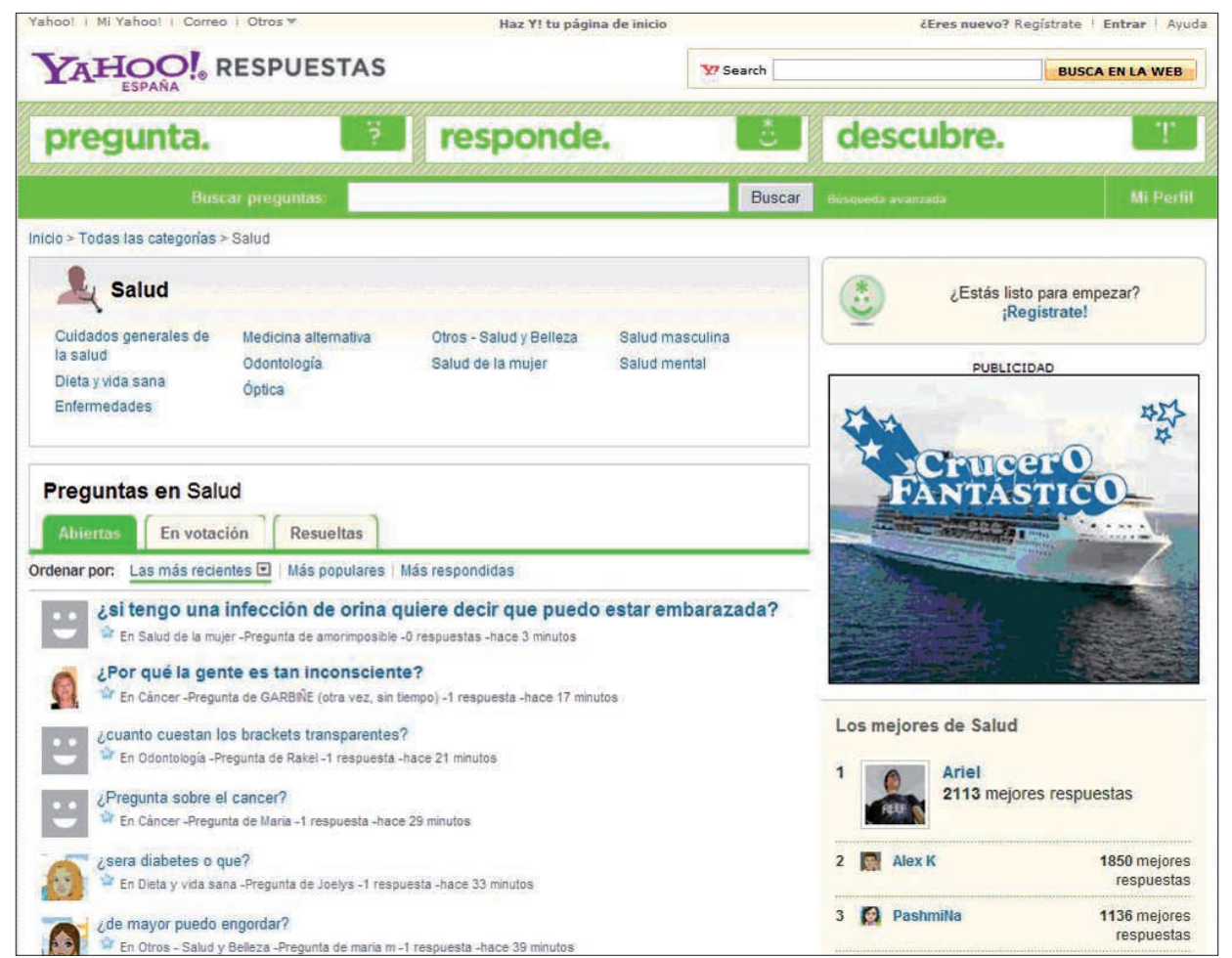

Figura 6. Foro sobre salud en la web de Yahoo en Estados Unidos, WebMD Health es el número uno en esta categoría de contenidos con 17,3 millones de visitas en julio de 2008. Everyday Health, con 14,7 millones de visitas, Revolution Health Network con 11,3 millones, y AOL Health con 11,1 millones son los competidores directos. La clave del crecimiento de estos sitios web de información médica y sanitaria se debe, por un lado, a su asociación reciente con sitios más pequeños y, por otro, a la realización de adquisiciones estratégicas que han permitido su viabilidad. Se habla, por tanto, de más de $50 \mathrm{mi}-$ llones de usuarios de contenidos médicos informativos sólo en Estados Unidos, toda una oportunidad para impulIncluso se podría considerar el engaño al navegante por parte de algún proveedor interesado.

http://es.answers.yahoo.com/dir/index;_ylt $=A j L j t X H$ mjeJ_H8SZpull5q3dHXRG;_ylv=3?sid=396545018\& link=open

\section{El futuro: movilidad para todos}

Las perspectivas de Google Health para los próximos años se abren también en la plataforma de dispositivos móviles. En aparatos de telefonía como el iPhone de Apple es posible, por ejemplo, conectar con la información clínica alojada en el servidor de Google. La aplicación gratuita denominada Health Cloud o Cloud PHR (figura 7), una vez instalada en este teléfono táctil, funciona generando información personalizada y estructurada y cuenta con la supervisión de profesionales de la medicina. Igualmente debe reseñarse que Google Health se basa en gran medida en las aplicaciones de su colaborador Anvita, cuya tecnología permite al paciente conocer las interacciones de los fármacos y sus posibles incompatibilidades, y al mismo tiempo le ofrece datos médicos de relevancia. En definitiva, todas estas innovaciones responden a una estrategia de comunicación interactiva que las administraciones sanitarias y las mismas empresas de tecnología del sector también han elaborado puntualmente por su cuenta.

http://snosrap.com/cloudphr/?r=1\&p http://www.google.com/intl/es/health/about/partners/ anvitahealth.html

Según datos de Comscore Media Metrix (tabla 1)², sar técnicas de autogestión de información médica.

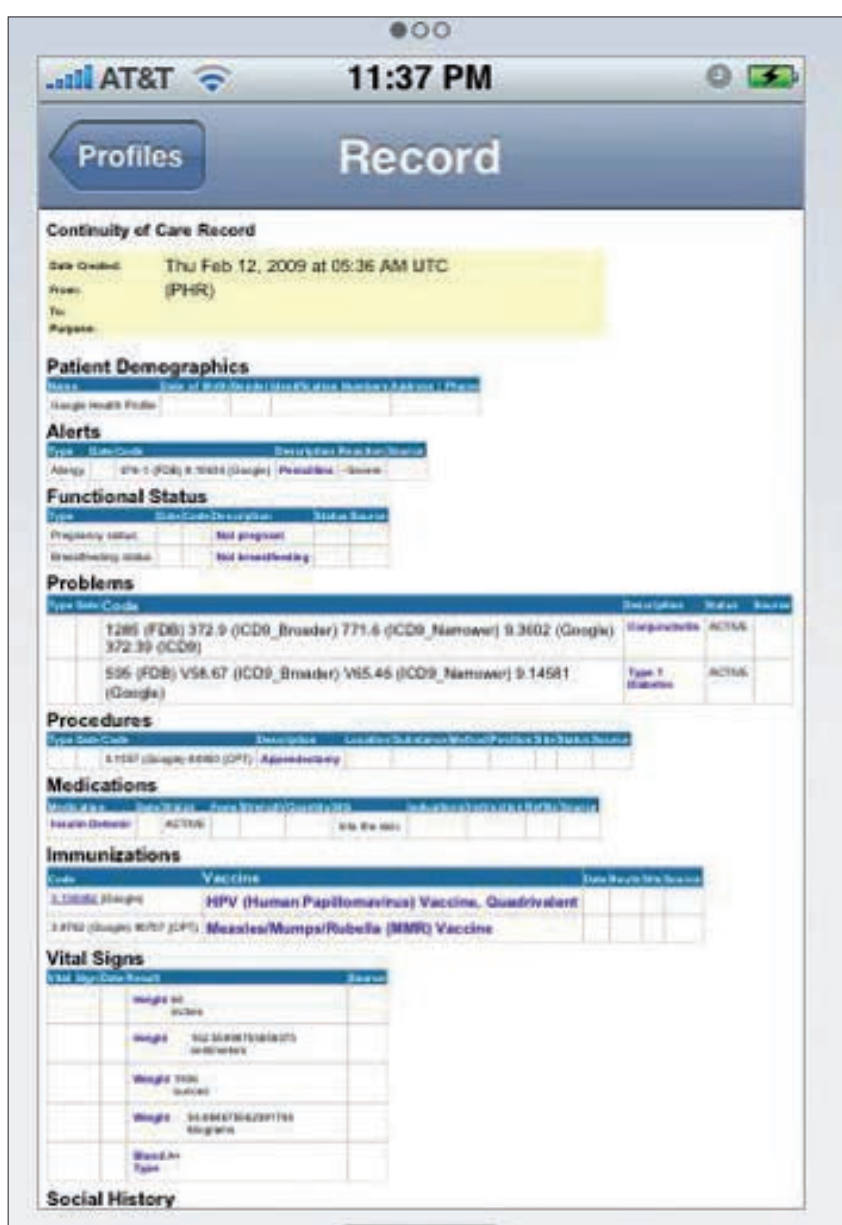

Figura 7. Health Cloud permite acceder a Google Health mediante un IPhone de Apple 


\begin{tabular}{|l|r|r|r|}
\hline \multicolumn{3}{|c|}{$\begin{array}{c}\text { Top } 10 \text { health information sites by unique visitors } \\
\text { July } 2008 \text { vs. July } 2007\end{array}$} \\
Total U.S. - Home/Work/University locations \\
Source: ComScore Media Metrix \\
\hline & \multicolumn{3}{|c|}{ Total unique visitors } \\
\cline { 2 - 4 } & $\begin{array}{r}\text { Julio } \\
\mathbf{2 0 0 7}\end{array}$ & $\begin{array}{c}\text { Julio } \\
\mathbf{2 0 0 8}\end{array}$ & $\begin{array}{c}\text { \% } \\
\text { change }\end{array}$ \\
\hline Total internet audience & 180.078 & 189.134 & 5 \\
\hline Health information source & 56.865 & 69.008 & 21 \\
\hline WebMD Health & 16.829 & 17.277 & 3 \\
\hline Everyday Health & 9.009 & 14.703 & 63 \\
\hline Revolution Health Network & 4.014 & 11.329 & 182 \\
\hline AOL Health & 5.913 & 11.095 & 88 \\
\hline About.com Health & 6.947 & 8.682 & 25 \\
\hline Yahoo! Health & 7.445 & 8.496 & 14 \\
\hline MSN Health & 8.833 & 7.813 & -12 \\
\hline NIH.Gov & 8.545 & 7.315 & -14 \\
\hline Righthealth.com & 2.424 & 6.160 & 154 \\
\hline Quality Health Network & N/A & 5.822 & N/A \\
\hline
\end{tabular}

Tabla 1. Páginas de salud de los EUA más visitadas según Comscore Media Metrix

Insistiendo en el futuro de la información móvil, casi dos tercios de los usuarios de internet en Estados Unidos emplearían los servicios de salud móviles si estuvieran disponibles (CTIA-The Wireless Association; Harris Poll Interactive, 2009). La mayoría de los clientes los utilizarían como complemento, aunque el $23 \%$ de los encuestados es partidario de sustituir las visitas presenciales al facultativo por una atención recibida empleando dispositivos móviles, siempre que sea posible y que les permita también conocer el estado de su salud, recomendaciones clínicas y plan de atención. Las exploraciones y las consultas son el uso más popular de salud móvil, seguido de los cuidados para la salud, la vigilancia epidemiológica y los recordatorios médicos. Es, en consecuencia, la nueva pantalla a la que todos tendrán que adaptarse con aplicaciones, contenidos de servicio ajustados y documentación médica de máximo rigor y utilidad.

\section{Notas}

1. La Health on the Net Foundation es una organización no gubernamental suiza que fomenta la difusión online de información de salud de calidad. Este organismo es el responsable de la acreditación Hon Code. http://www.hon.ch/homel_sp.html.

2. Resultados de medición hechos públicos por Comscore Media Metrix mediante una nota de prensa.

http://www.comscore.com/es//Press_Events/Press_Releases/2008/09/Top_ Internet_Healths_Sites.

\section{Bibliografía}

Brownstein, John; Freifeld, Clark; Madoff, Lawrence. "Digital disease detection - Harnessing the web for public health surveillance". The New England journal of medicine, 2009, mayo, v. 360, n. 21, pp. 2153-2157.

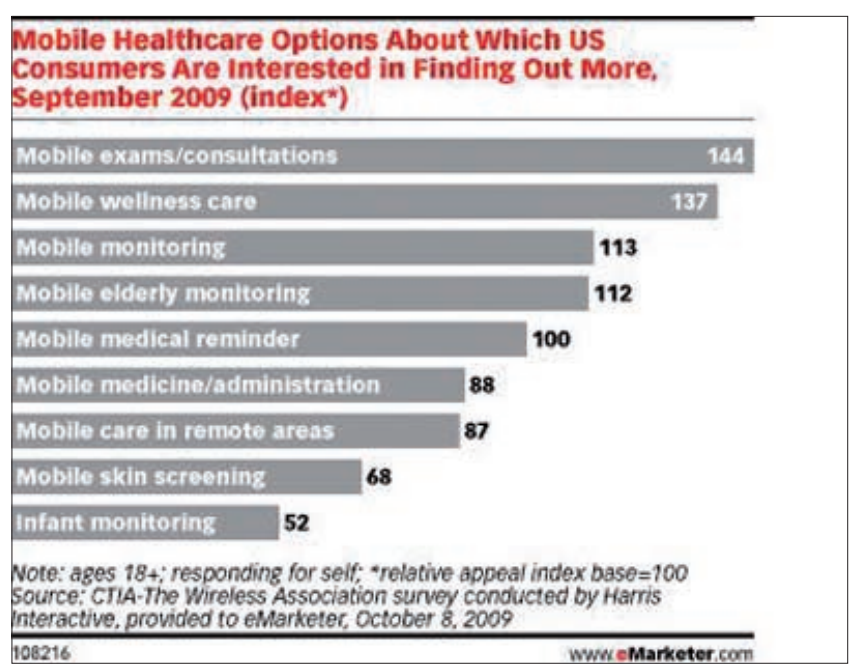

Figura 8. Usos de salud móvil según CTIA-The Wireless Association y Harris Poll Interactive

Carneiro, Herman; Mylonakis, Eleftherios. "Google trends: a web-based tool for real-time surveillance of disease outbreaks". Clinical infectious diseases, 2009, noviembre, v. 49, n. 10, pp. 1557-1564.

CTIA-The Wireless Association; Harris Poll Interactive. Mobile healthcare options about which US consumers are interested in finding out more. Washington, CTIA-The Wireless Association y Harris Poll Interactive, 2009.

Departamento de Estudios Sociales y de Opinión Pública de la Fundación BBVA. II Estudio sobre internet en España. Madrid, Fundación BBVA, 2008.

Ginsberg, Jeremy; Mohebbi, Matthew; Patel, Rajan; Brammer, Lynnette; Smolinski, Mark; Brilliant, Larry. "Detecting influenza epidemics using search engine query data". Nature, 2009, febrero, v. 457, pp. 1012 1014 .

Guanyabens, Joan. "La recepta electrònica a Catalunya dins el marc global de la política de les TIC". En Jornada de la receta electrónica en el contexto de Europa, 2008.

http://www10.gencat.net/catsalut/archivos/jornades/recepta1/Joan_ Guanyabens.pdf

Instituto Nacional de Estadística. Encuesta sobre equipamiento y uso de tecnologías de la información y comunicación en los hogares. Madrid, Instituto Nacional de Estadística, 2009.

Lupiáñez-Villanueva, Francisco. Internet, salud y sociedad. Análisis de los usos de internet relacionados con la salud en Catalunya. Tesis doctoral defendida en la Universidat Oberta de Catalunya (Barcelona) en 2009. http://www.tdr.cesca.es/TESIS_UOC/AVAILABLE/TDX-0528109-092039// tesis_flupianez_21_11_08.pdf

Moyá-Garrido, María-Natividad. Internet, salud y ciudadanía. Sevilla: Agencia de Evaluación de Tecnologías Sanitarias, 2009, ISBN: 978-84-6922815-9.

http://www.juntadeandalucia.es/salud/servicios/contenidos/aetsa/pdf/ Imternet_y_salud_def.pdf

Pew \& American Life Project; California HealthCare Foundation. The social life of health information. Washington, 2009.

Tang, Paul; Lee, Thomas. "Your doctor's office or the internet? Two paths to personal health records". The New England journal of medicine, 2009, marzo, v. 360, n. 13, pp. 1276-1278.

Toni González-Pacanowski, Pablo Medina-Aguerrebere. Universidad de Navarra.

tonigonza@telefonica.net

pamedina@unav.es 